\title{
Neonatal iliopsoas abscess presenting with transient cyanosis of a single extremity: a case report and review of the literature
}

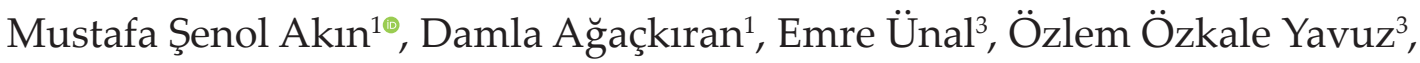 \\ Şule Yiğgit² \\ ${ }^{2}$ Division of Neonatology, ${ }^{1}$ Department of Pediatrics and ${ }^{3}$ Department of Radiology, Hacettepe University Faculty of Medicine, Ankara, \\ Turkey.
}

\begin{abstract}
A newborn baby with an unusual complaint of transient left leg cyanosis during crying, who was diagnosed with a iliopsoas abscess is presented. Newborn cases diagnosed with an iliopsoas abscess in the English literature are summarized and differences in clinical presentations are discussed.
\end{abstract}

Key words: transient single extremity cyanosis, iliopsoas abscess, newborn.

The iliopsoas abscess is rare in children, while it is highly rare in the newborn period. To date, 24 cases with iliopsoas abscess occurring in the neonatal period have been reported in the literature (Table I). The etiological factors in most of the cases have not been identified. Birth trauma and local hemorrhage in the iliopsoas muscle have been accused as leading factors for abscess formation. The most common symptoms of iliopsoas abscess in newborns are pain during hip movement and swelling of thigh. We present here a case who presented with an unusual symptom; transient left leg cyanosis becoming evident during crying.

\section{Case Report}

The patient was a $3650 \mathrm{gr}$ female baby, born to a 29 years old mother at 37 weeks and 5 days of gestation with cesarean section. Pregnancy was uneventful. However, the baby was taken to the neonatal intensive care unit (NICU) on the first day of life due to sub-febrile fever. During

$凶$ Mustafa Şenol Akın

mustafasenolakin@gmail.com

Received 28th December 2018; revised 25th February 2019; accepted 29th May 2019
NICU care antibiotic treatment was not given since acute phase reactants and blood culture were negative and fever did not persist.

The patient was discharged on the 5th day. After discharge the mother noticed that her baby had cyanosis on her left leg when she cried. On $13^{\text {th }}$ day of life she was admitted to our hospital due to increase in cyanosis during crying and limitation of left hip movement. Physical examination revealed nothing except, slight limitation of left hip movement and slight swelling of thigh. The left thigh circumference was measured as $18 \mathrm{~cm}$ while the right thigh circumference was $17 \mathrm{~cm}$. Pulse oxymeter showed no difference in oxygen saturation between right and left leg when the baby was peaceful. However left legs oxygen saturation decreased to $60 \%$ while baby was crying and cyanosis occurred on the left leg while the right leg saturation was still $100 \%$. Cyanosis disappeared and left leg saturation increased when the baby calmed down. Treatment of vancomycin and amikacin were given considering septic arthritis.

Hemogram resulted as follows; hemoglobin $12.4 \mathrm{~g} / \mathrm{dl}$, white blood cell count 17,700/ $\mathrm{mm}^{3}$, immature total ratio 0.31 , platelet 688,000 / 
$\mathrm{mm}^{3}$, c-reactive protein $2,5 \mathrm{mg} / \mathrm{dl}$, complete urinalysis was normal and blood culture was negative. Echocardiography was normal.

Doppler ultrasonography (US) showed normal arterial and venous blood flow of the lower extremity on both sides, but it was noticed that femoral artery and vein blood flow was interrupted temporarily on the left side when the baby cried. There was no thrombus or occlusion in the vascular structures. Superficial ultrasound revealed cutaneous and subcutaneous edema findings and a few small reactive lymph nodes on the left inguinal and femoral region suggesting infectious or inflammatory pathologies. Left hip joint was normal on US. Abdominal US showed asymmetrical thickness of left psoas muscle. For detail evaluation magnetic resonance imaging (MRI) was done without contrast. Abdominal MRI revealed multifocal abscess in iliopsoas muscle and soft tissue inflammation in the inguinal region (Fig. 1ab). We think that when intraabdominal pressure increased with crying, the left iliopsoas abscess compressed the iliac vasculature with mass effect and disturbed the blood flow and as a result, her left leg became cyanotic. The abscess was aspirated with a 19-gauge needle under US guidance by the interventional radiology department. Methicillin-resistant staphylococcus aureus was determined in the pus sent from the abscess. After the abscess was drainage, the patient's symptoms disappeared within the same day. The patient was discharged, after receiving vancomycin amikacin therapy for 4 weeks. Permission was obtained from the parents for publication of this case and informed consent was obtained from the family.

\section{Discussion}

The abscess of iliopsoas is rarely seen in the newborn period. We found 24 cases in the literature. These cases are summarized in Table I. Hospital admission usually occurs after the 2nd week of life, so it is thought that abscess formation occurs in the postnatal period. Our case applied on the 13th day of life. Six out of 24 cases in the literature had a fever and most of the time there is no symptom of fever as in our case. Of the 24 cases in the literature, 15 were male, the male / female ratio was 1.6, and if we include our case, the rate is 1.5. The most common symptom is pain and swelling on the lower extremity. In addition, inguinal region lymph nodes are palpable on the relevant side. Our case is the first case which was presented with cyanosis of a single leg while crying.

In very few cases, white blood cell count increase was determined. As in our case, there has been
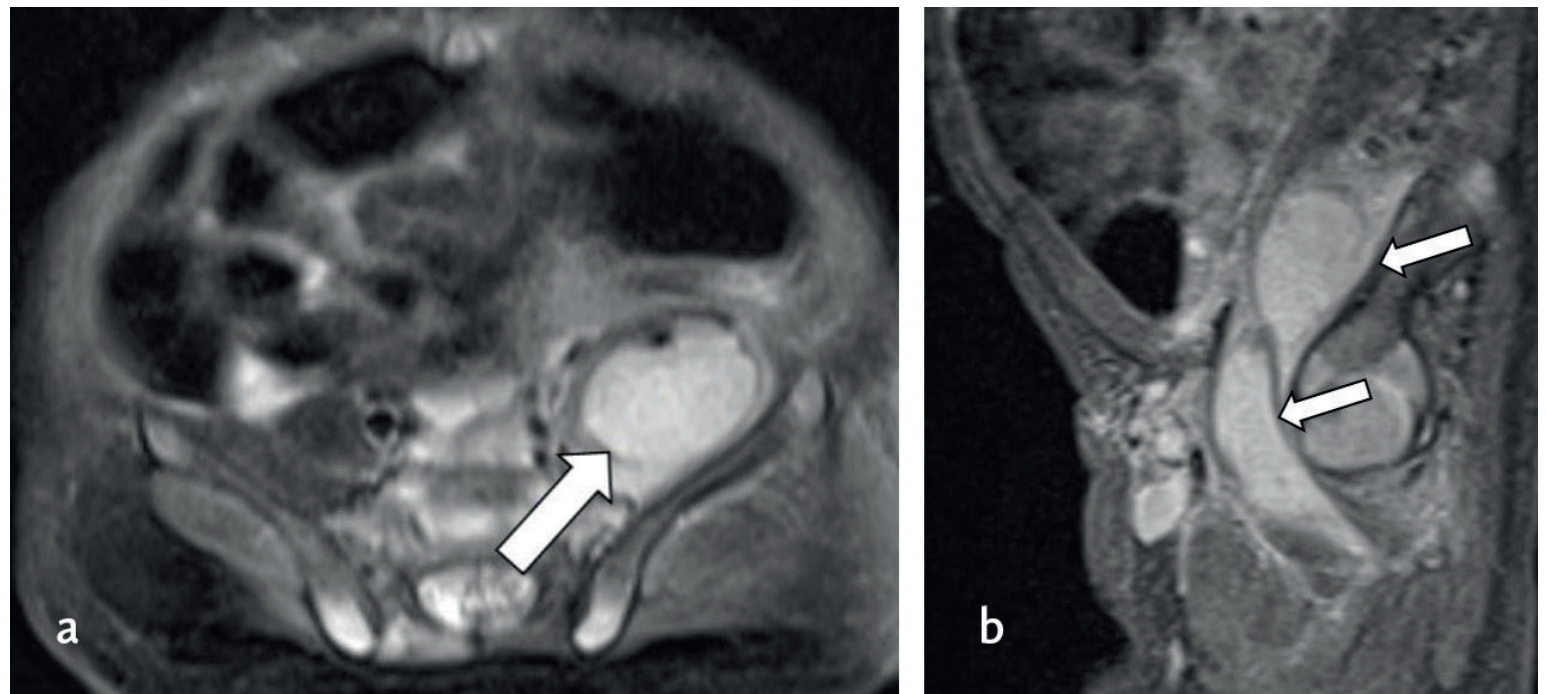

Fig. 1. Axial (a) and sagittal (b) T2-weighted images of MRI shows multiple abscess in iliopsoas muscle (arrows). 


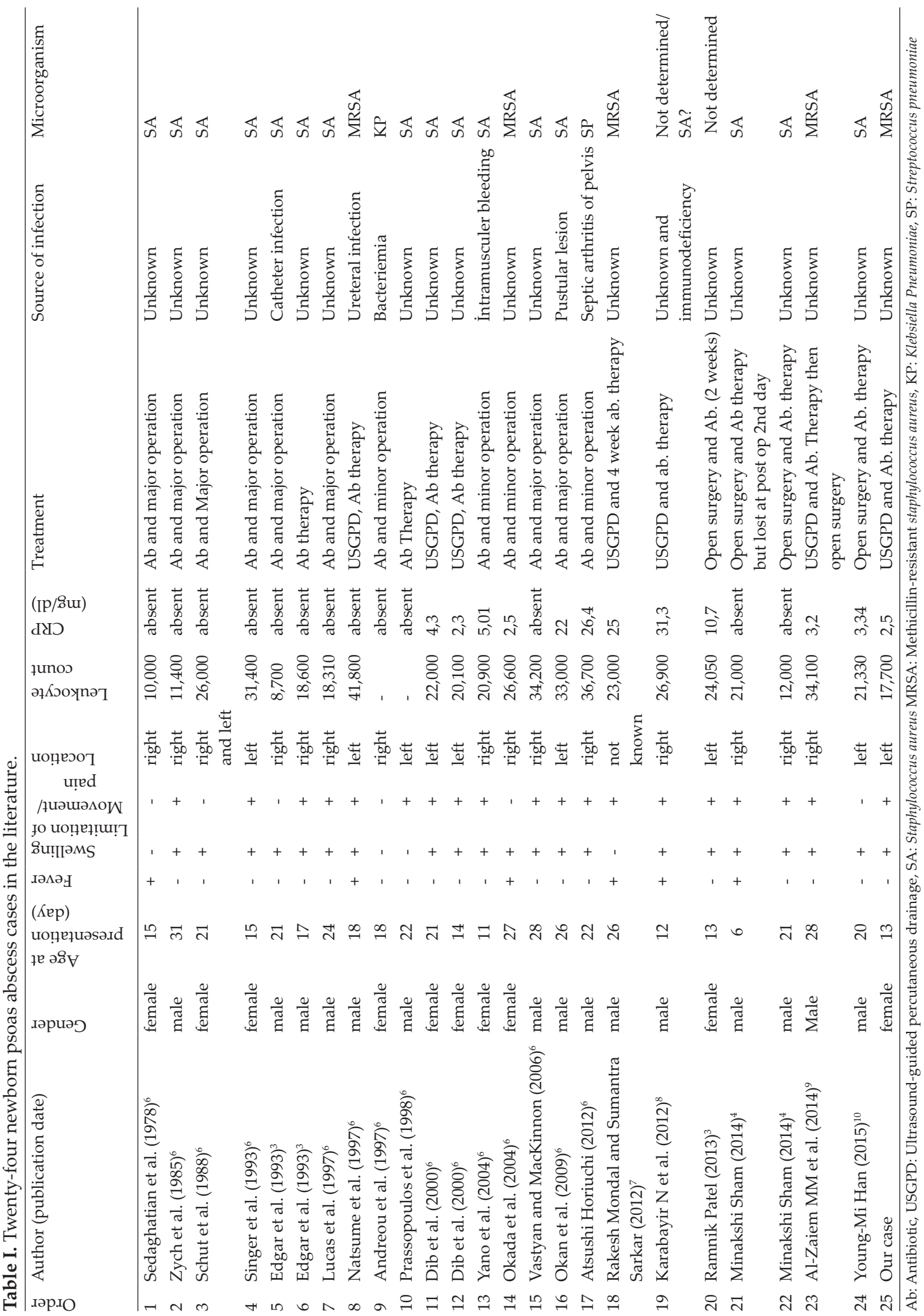


a slight increase in the acute phase reactors and an increase in white blood cell count has not been detected in most of the cases. Although US and computerized tomography (CT) have been used for diagnosis in most of the cases; MRI was used in our case after US evaluation. Compared to CT, advantages of MRI are no radiation exposure and high soft tissue resolution.

The first step of the treatment of abscess is drainage of the abscess. Usually antibiotic therapy alone is not sufficient for treatment. However, two cases in the literature were treated with only antibiotic therapy and their abscesses retracted with only antibiotic therapy. ${ }^{1,2}$ In the literature, as in our case, US guided needle drainage was used at six cases and no additional open surgery was needed. There have been other treatment options ranging from a small cut made in the inguinal region to large open surgery. It should be debated which treatment method is better. However, it is suggested that the least invasive method should be tried first during the neonatal period and if it fails, more invasive methods should be performed.

Microorganism could be produced from blood cultures in a few cases while it is mostly produced from pus culture. Staphylococcus aureus was the most frequently isolated microorganism from iliopsoas abscess. The microorganisms were Staph. Aureus in 16 cases, methicillinresistant Staphylococcus aureus (MRSA) in 5 cases with our case, Klebsialla pneumonia in one case, Staph. Hominis in one case and Streptococcus pneumonia in one case. In only one of these cases was no agent isolated. As in our case, the presence of elevated CRP, pain during hip movement and swelling of the thigh were the most common symptoms leading doctors to decide to start antibiotics. Septic arthritis was the most common preliminary diagnosis. It is recommended that antibiotic therapy should be given for 4 to 6 weeks, depending on the patient's clinic and doctor's assessment.

The etiological factors leading to abscess formation in the iliopsoas in neonates have not been identified. Birth trauma and possible intramuscular hemorrhage are accused reasons for abscess formation. Due to the weak immune system of newborns, a possible microorganism in the skin flora or gastrointestinal flora can reach the damaged region by hematologic route and may form an abscess there. Intramuscular injections for prophylactic treatments may cause the spread of skin or hospital flora. In our case, there may be healthcare-associated infection. MRSA detection and hospitalization history support this.

In the literature, one case was lost due to sepsis two days after open drainage of iliopsoas abscess but the prognosis of other patients with early diagnosis and abscess drainage is quite good. ${ }^{3}$ None of the cases in the literature report sequelae or re-abscess formation occurring in the same region.

Transient local cyanosis is not a common finding in newborns. We found two cases with local cyanosis in the literature. One case's symptom was left leg cyanosis due to large bladder compression as a complication of circumcision. ${ }^{4}$ The other case presented with upper left limb cyanosis and was diagnosed with raynaud phenomenon. ${ }^{5}$ However in both cases cyanosis were not transient.

Our patient is the only case in the literature that presented with transient left leg cyanosis during crying and was discharged completely healthy after 4 weeks of amikacin and vancomycin treatment.

\section{REFERENCES}

1. Edgar EA, Schlesinger AE, Royster RM, Deeney VFX. Ilo-psoas abscess in neonates. Pediatr Radiol 1993; 23: 51-52.

2. Prassopoulos PK, Giannakopoulou CA, Apostolaki EG, Charoulakis NZ, Gourtsoyiannis NC. Primary ilio-psoas abscess extending to the thigh in a neonate: US, CT and MR findings. Pediatr Radiol 1998; 28: 605-607.

3. Sham M, Singh D. Neonatal ilio-psoas abscess: report of two cases. J Neonatal Surg 2014; 3: 4. 
4. Arnon R, Zecharia A, Mimouni M, Merlob P. Unilateral leg cyanosis: an unusual complication of circumcision. Eur J Pediatr 1992; 151: 716.

5. Sharathkumar AA, Castillo-Caro P. Primary Raynaud's phenomenon in an infant: a case report and review of literature. Pediatr Rheumatol Online J 2011; 9: 16.

6. HoriuchiA, Kameoka K, Kuwabara J, et al. Neonatal iliopsoas abscess. Pediatr Int 2012; 54: 712-714.

7. Mondal R,Sarkar S. Spondylodiscitis with primary psoas abscess in a neonate. Indian Pediatr 2012; 49: 681.
8. Karabayir N, Turel O, Aydogmus C, Hatipoglu N, Hocaoglu A, Adal E. Iliopsoas abscess in the neonate with immunodeficiency. Pediatr Int 2012; 54: 439-440

9. Al-Zaiem MM, Bajuifer SJ, Fattani MO, Al-Zaiem FM. Bilateral iliopsoas abscess associated with right hip septic arthritis in a neonate. Saudi Med J 2014; 35: 743-746.

10. Han YM, Kim AY, Lim RK, et al. Neonatal iliopsoas abscess: the first Korean case. J Korean Med Sci 2015; 30: 1203-1206. 